\title{
Quark anomalous magnetic moment and its effects on the $\rho$ meson properties
}

\author{
Zanbin Xing, ${ }^{1, *}$ Khépani Raya $\oplus^{1,2,3, \dagger}$ and Lei Chang ${ }^{1, \$}$ \\ ${ }^{1}$ School of Physics, Nankai University, Tianjin 300071, China \\ ${ }^{2}$ Departamento de Física Teórica y del Cosmos, Universidad de Granada, E-18071, Granada, Spain \\ ${ }^{3}$ Instituto de Ciencias Nucleares, Universidad Nacional Autónoma de México, \\ Apartado Postal 70-543, CdMx 04510, Mexico
}

(Received 11 July 2021; accepted 31 August 2021; published 28 September 2021)

\begin{abstract}
A symmetry-preserving treatment of mesons, within a Dyson-Schwinger and Bethe-Salpeter equations approach, demands an interconnection between the kernels of the quark gap equation and meson BetheSalpeter equation. Appealing to those symmetries expressed by the vector and axial-vector Ward-GreenTakahashi identitiges (WGTI), we construct a two-body Bethe-Salpeter kernel and study its implications in the vector channel; particularly, we analyze the structure of the quark-photon vertex, which explicitly develops a vector meson pole in the timelike axis and the quark anomlaous magnetic moment term, as well as a variety of $\rho$ meson properties: mass and decay constants, electromagnetic form factors, and valencequark distribution amplitudes.
\end{abstract}

DOI: $10.1103 /$ PhysRevD.104.054038

\section{INTRODUCTION}

Quantum chromodynamics (QCD) is regarded as the underlying theory of the nuclear strong interactions, and so hadron physics. Even though its Lagrangian is apparently simple, high-level complexity phenomena take place, such as quark-gluon confinement and the emergence of hadron masses (EHM) [1]. The Dyson-Schwinger equations (DSE) formalism has proven to be a very robust approach to QCD in the continuum [2,3], capable of taming its nonperturbative character. Supplemented by the bound-state Bethe-Salpeter (BS) and Faddeev equations [4,5], the DSE formalism becomes an ideal platform for the calculation of hadron masses and several structural properties; see, for example Refs. [6-9]. The derivation of QCD's DSEs do not require any assumptions on the running coupling, therefore, both perturbative and nonperturbative facets of the strong interactions can be addressed within this formalism. At the same time, the DSE approach is not restricted to a certain domain of current quark masses [9-12]. Moreover, hadron observables can be traced down to fundamental pieces, namely propagators and vertices, hence maintaining a clear connection to QCD's fundamental degrees of freedom, quarks and gluons [13-16]. The structure of the DSEs is such that any $n$-point Green function is related to at least one higher order function, therefore yielding an infinite set of coupled

\footnotetext{
*xingzb@mail.nankai.edu.cn

tkhepani@nankai.edu.cn

"leichang@nankai.edu.cn
}

integral equations [2]. In order to arrive at a tractable problem, a sensible truncation is needed; thus, systematicity and symmetry principles become imperative [17-19]. Historically, Ward-Green-Takahashi identities (WGTI) [20-22] have been crucial in symmetry-preserving studies of hadron properties. Such relations ensure, among other things, current-conservation and the appearance of Goldstones modes in connection with dynamical chiral symmetry breaking (DCSB) $[18,19]$. Furthermore, WGTI impose relationships between propagators and vertices, as well as constraints between the kernels of the one-body and two-body problems $[23,24]$. Nevertheless, the mathematical form of the one-body and two-body kernels (quark self energy and BS equation kernels), which satisfy such consistency relations, is not always unique [25]. Taking advantage of this fact, we derive a modified version of the so called rainbow-ladder (RL) truncation. Subsequently, within a contact interaction (CI) model, we analyze its impact on the structure of the quark-photon vertex (QPV) and $\rho$ meson properties, as derived from their corresponding Bethe-Salpeter equations. More precisely, we shall expose how, in addition to the $\rho$ meson pole in the timelike axis, the structure of the QPV develops an anomalous magnetic moment (AMM) term. It is known that DCSB generates an anomalous chromonagnetic moment (ACM) for dressed light-quarks, which is large at infrared momenta and generates an electromagnetic moment with commensurate size but opposite sign [26,27]. Thus, it is a highly desirable feature for the QPV, exhibited quite transparently in our present approach. Its impact on the structural properties of hadrons is put in manifest when studying 
electromagnetic form factors (EFF): while the elastic EFF of spin- 0 mesons is unaltered by the AMM piece, the latter displays from mild to notorious impact on EFF involving spin-1 mesons (and baryons as well) [28-30].

The manuscript is organized as follows: Sec. II introduces aspects of constructing symmetry-preserving truncations of quark DSE and meson BS equation, based upon vector and axial-vector WGTIs. In Sec. III, we present the CI model within the RL truncation and its modified version (MRL). The mass spectrum of light-mesons $\{\pi, \rho\}$ is included to compare and contrast. Section IV focuses on the derivation and structure of the QPV. Subsequent sections capitalize on $\rho$ meson structural properties: EFFs of the $\rho$ meson are discussed in Sec. V, while its valence-quark distribution amplitudes (PDAs) are derived in Sec. VI. Conclusions and final remarks are presented in Sec. VII.

\section{SYMMETRY-PRESERVING TRUNCATIONS}

Within the DSE approach, the properties of valence quark/antiquark bound-states are encoded within solutions of its BS equation. Mesons appear as poles in the corresponding inhomogeneous BS equation [23,24], which takes the form [23,24]:

$$
\Gamma_{H}(p ; P)=\tilde{\gamma}_{H}+\int_{q} K^{(2)}(q, p ; P) \chi_{H}(q ; P),
$$

where $\chi_{H}(q ; P)=S\left(q_{+}\right) \Gamma_{H}(q ; P) S\left(q_{-}\right)$denotes the BS wave function, such that: $\Gamma_{H}(q ; P)$ corresponds to the BS amplitude of a meson $H$, whose specific structure in terms of Dirac matrices depends on its quantum numbers; $S\left(q^{ \pm}\right)$represent the quark and antiquark propagators. The two particle irreducible quark/antiquark scattering kernel is denoted by $K^{(2)}(q, p ; P)$; and $\tilde{\gamma}_{H}$, as the BS amplitude, is defined by a specific combination of Dirac matrices that specify the $J^{P C}$ channel $\left(\tilde{\gamma}_{H}=\gamma_{\mu}\right.$ for the vector vertex and $\tilde{\gamma}_{H}=\gamma_{5} \gamma_{\mu}$ for the axial-vector vertex). Herein, we use the notation $\int_{q}$ to refer to four dimensional Euclidean integral, regularized in a Poincaré covariant manner. Finally, the kinematics is defined as follows: $P$ is the total momentum of the quark/antiquark system; $q_{+}=q+\eta P$ and $q_{-}=q-(1-\eta) P$, with $\eta \in[0,1]$ defining the relative momentum (in a Poincaré covariant framework, no single observable depends on $\eta$ ). The fully dressed quark propagator obeys a DSE of the form:

$$
S^{-1}(p)=\left[S^{(0)}(p)\right]^{-1}+\int_{q} K^{(1)}(q, p) S(q),
$$

where $S^{(0)}(p)=\left[i \gamma \cdot p+m^{\mathrm{bm}}\right]^{-1}$ corresponds to the bare quark propagator, with a Lagrangian current quark mass $m^{\mathrm{bm}}$, and $K^{(1)}(q, p)$ the one-body kernel. The above equation is often referred to as gap equation. In both Eqs. (1), (2), and throughout the rest of the manuscript, we suppress all remormalization constants, as well as color and flavor indices, for notational convenience.

As has been pointed out in the Introduction, a practical way to construct and relate $K^{(1)}$ and $K^{(2)}$, relies upon WGTIs. The chiral limit vector and axial vector WGTIs take the form:

$$
\begin{gathered}
i P_{\mu} \Gamma_{\mu}(k ; P)=S^{-1}\left(k_{+}\right)-S^{-1}\left(k_{-}\right) . \\
P_{\mu} \Gamma_{5 \mu}(k ; P)=S^{-1}\left(k_{+}\right) i \gamma_{5}+i \gamma_{5} S^{-1}\left(k_{-}\right),
\end{gathered}
$$

where $\Gamma_{\mu}$ and $\Gamma_{5 \mu}$ are the vector and axial-vector vertices. Now consider the gap equation in QCD [2]:

$S^{-1}(p)=\left[S^{(0)}(p)\right]^{-1}+\frac{4}{3} g^{2} \int_{q} D_{\mu \nu}(p-q) \gamma_{\mu} S(q) \Gamma_{\nu}(p, q)$,

where $g$ is the Lagrangian coupling constant; $D_{\mu \nu}$ and $\Gamma_{\nu}$ are the fully dressed gluon propagator and quark-gluon vertex (QGV), respectively. Thus we can identify

$$
K^{(1)}(q, p)=\frac{4}{3} g^{2} D_{\mu \nu}(p-q) \gamma_{\mu} \otimes \Gamma_{\nu}(q, p) .
$$

If we restrain ourselves to the tree-level QGV $\Gamma_{\nu} \rightarrow \gamma_{\nu}$, hence neglecting all the rich structure that the fully-dressed vertex might have $[27,31,32]$, and replace the gluon propagator by an effective one, $D_{\mu \nu} \rightarrow D_{\mu \nu}^{\text {eff }}$, to compensate the missing pieces in $\Gamma_{\nu}[33,34]$, the one-body kernel becomes

$$
K^{(1)}(q, p)=\frac{4}{3} g^{2} D_{\mu \nu}^{\mathrm{eff}}(p-q) \gamma_{\mu} \otimes \gamma_{\nu}
$$

The two-body kernel $K^{(2)}$ can be obtained by combining Eqs. (1)-(4) and Eq. (7), which yields

$$
\begin{aligned}
& \int_{q} K^{(2)}(q, p ; P)\left[S\left(q_{+}\right)-S\left(q_{-}\right)\right] \\
& \quad=-g^{2} \frac{4}{3} \int_{q} D_{\mu \nu}^{\mathrm{eff}}(p-q) \gamma_{\mu}\left[S\left(q_{+}\right)-S\left(q_{-}\right)\right] \gamma_{\nu},
\end{aligned}
$$

$$
\begin{aligned}
& \int_{q} K^{(2)}(q, p ; P)\left[S\left(q_{+}\right) \gamma_{5}+\gamma_{5} S\left(q_{-}\right)\right] \\
& \quad=-g^{2} \frac{4}{3} \int_{q} D_{\mu \nu}^{\mathrm{eff}}(p-q) \gamma_{\mu}\left[S\left(q_{+}\right) \gamma_{5}+\gamma_{5} S\left(q_{-}\right)\right] \gamma_{\nu} .
\end{aligned}
$$

Then the simplest choice that satisfies the vector and axialvector WGTIs symmetry constraints is

$$
K^{(2)}(q, p ; P)=-\frac{4}{3} g^{2} D_{\mu \nu}^{\mathrm{eff}}(p-q) \gamma_{\mu} \otimes \gamma_{\nu}=-K^{(1)}(q, p ; P) .
$$


Such kernels define the RL truncation: $K^{(1)}$ refers to the rainbow part, while $K^{(2)}$ corresponds to the ladder piece. Notably, this simple choice is sufficient to ensure the appearance of pions as Goldstone bosons of DCSB $[18,19]$, while also being a sensible approximation to compute its structural properties $[13,14,35]$.

It is worth pointing out that the solution to Eqs. (8)-(9) is not unique. In fact, given the rainbow approximation for the one body problem, it is possible to derive a fully consistent symmetry-preserving two body kernel that extends beyond the RL truncation, as it is discussed in Refs. [25,36]. In addition to the ladder part, other terms of the form

$K^{(2)}(q, p ; P)=\tilde{D}^{j}(q, p) \tilde{\Gamma}_{j}(q, p) \otimes \tilde{\Gamma}_{j}(q, p) F^{j}(q, p)$,

can be added to the two-body kernel [37]. Herein, $F^{j}$ are Lorentz invariant scalar functions, $\tilde{\Gamma}_{j}$ are different combinations of Dirac matrices, and $\tilde{D}^{j}$ are tensor structures that might be needed to contract Lorentz indices. The particular choices $\tilde{\Gamma}_{j}(q, p)=\left\{\mathbb{I}, \gamma_{5}, i / \sqrt{6} \sigma_{\mu \nu}\right\}$ and $\tilde{D}^{j}(p, q) F^{j}(p, q) \rightarrow \xi \tilde{D}$, the latter being reduced to a simple constant, preserve the consistency constraints and so Eqs. (8)-(9). Thus, the inhomogeneous BS equation becomes

$$
\begin{aligned}
\Gamma_{H}(q ; P)= & \tilde{\gamma}_{H}-\frac{4}{3} g^{2} \int_{q} D_{\mu \nu}^{\mathrm{eff}}(p-q) \gamma_{\mu} \chi_{H}(q ; P) \gamma_{\nu} \\
& +\xi \tilde{D} \int_{q} \tilde{\Gamma}_{j} \chi_{H}(q ; P) \tilde{\Gamma}_{j} .
\end{aligned}
$$

The product $\xi \tilde{D}$ sets the strength of the nonladder (NL) term. In the next section we shall discuss about the truncation herein derived, Eq. (12), within a vector-vector symmetry-preserving contact interaction (CI) model of QCD $[28,38,39]$.

\section{CONTACT INTERACTION MODEL}

Let us recall the quark gap equation in the RL truncation:

$$
S^{-1}(p)=\left[S^{(0)}(p)\right]^{-1}+\frac{4}{3} \int_{q} g^{2} D_{\mu \nu}^{\mathrm{eff}}(p-q) \gamma_{\mu} S(q) \gamma_{\nu}
$$

Clearly, the quark DSE decouples from the QGV and gluon DSEs. The only remaining ingredient is $D_{\mu \nu}^{\text {eff }}(p-q)$, the effective gluon propagator. This piece is supposed to compensate for all the missing pieces in the QGV $[33,34]$, often requiring an artificial enhancement in the infrared [32]. Thus we appeal to the illustrative CI model introduced in Refs. [38,39],

$$
g^{2} D_{\mu \nu}^{\mathrm{eff}}(p-q) \rightarrow \frac{1}{m_{G}^{2}} \delta_{\mu \nu}
$$

where $m_{G}=0.132 \mathrm{GeV}$ is an infrared mass scale. Besides preserving the relevant symmetries, the CI model typically yields semialgebraic expressions and captures the nonperturbative traits of QCD $[28,38,39]$. In addition, the CI model produces sensible results for the hadron mass spectrum [40-43], including tetraquarks [44], while also providing crucial benchmarks for many hadron structural properties [28-30,45-48]. The following sections are dedicated to illustrate some of the implications of the gluon model Ansatz from Eq. (14), in the RL and MRL truncations.

\section{A. Contact interaction in RL truncation}

In the CI model, the DSE for the quark propagator adopts the form

$$
S^{-1}(p)=\left[S^{(0)}(p)\right]^{-1}+\frac{4}{3 m_{G}^{2}} \int_{q} \gamma_{\mu} S(q) \gamma_{\mu},
$$

while the homogeneous meson BS equation is written as

$$
\Gamma_{H}(p ; P)=-\frac{4}{3 m_{G}^{2}} \int_{q} \gamma_{\mu} \chi_{H}(q ; P) \gamma_{\mu} .
$$

As it can be noticed, Eq. (15) possesses a quadratic divergence. A general solution adopts the form $S^{-1}(p)=$ $i \gamma \cdot p+M$, which exhibits a momentum independent mass function, $M$. The gap equation thus becomes

$$
M=m+\frac{M}{3 \pi^{2} m_{G}^{2}} \int_{0}^{\infty} d s \frac{s}{s+M^{2}} .
$$

Following standard literature, $[38,49]$, we then perform a proper time regularization ${ }^{1}$ :

$$
\frac{1}{s+M^{2}}=\int_{0}^{\infty} d \tau e^{-\tau\left(s+M^{2}\right)} \rightarrow \int_{\tau_{\mathrm{UV}}^{2}}^{\tau_{\mathrm{IR}}^{2}} d \tau e^{-\tau\left(s+M^{2}\right)} .
$$

The mass scale $\Lambda_{\mathrm{IR}}:=1 / \tau_{\mathrm{IR}}=0.24 \mathrm{GeV}$ guarantees confinement by ensuring the absence of quark production thresholds, while $\Lambda_{\mathrm{UV}}:=1 / \tau_{\mathrm{UV}}=0.905 \mathrm{GeV}$ represents an ultraviolet cutoff, setting the scale of all dimensioned quantities because the theory is non renormalizable. Therefore, the mass function can be obtained by solving

$$
M=m+\frac{M}{3 \pi^{2} m_{G}^{2}} C^{i u}\left(M^{2}\right),
$$

\footnotetext{
${ }^{1} \mathrm{~A}$ substraction scheme is also possible, as illustrated in Ref. [50].
} 


$$
\frac{C^{i u}\left(M^{2}\right)}{M^{2}}=\Gamma\left(-1, M^{2} \tau_{\mathrm{UV}}^{2}\right)-\Gamma\left(-1, M^{2} \tau_{\mathrm{IR}}^{2}\right),
$$

where $\Gamma(a, z)$ is the incomplete Gamma function.

Concerning the meson BS equation in the CI, Eq. (16), it is clear that a dependence on the relative momentum is forbidden by the interaction. Then, the pseudoscalar and vector meson BS amplitudes adopt the form

$$
\begin{aligned}
& \Gamma_{0^{-}}(P)=\gamma_{5}\left[i E_{0^{-}}(P)+\frac{\gamma \cdot P}{M} F_{0^{-}}(P)\right], \\
& \Gamma_{\mu}^{1^{-}}(P)=\gamma_{\mu}^{T} E_{1^{-}}(P)+\frac{1}{M} \sigma_{\mu \nu} P_{\nu} F_{1^{-}}(P),
\end{aligned}
$$

where $\gamma_{\mu}^{T}=\gamma_{\mu}-\frac{\gamma \cdot P}{P^{2}} P_{\mu}$. In a RL treatment of the CI, $F_{1^{-}}(P)=0$ (an analogous result holds for the axial-vector meson). Appealing to the WGTIs from Eqs. (3), (4), and contracting with $P_{\mu}$, one arrives at the chiral limit identities $\left(P^{2}=0=-m_{H}^{2}\right)$ :

$$
\begin{aligned}
M & =\frac{8 M}{3 m_{G}^{2}} \int_{q}\left[\frac{1}{q^{2}+M^{2}}+\frac{1}{(q-P)^{2}+M^{2}}\right], \\
0 & =\int_{q}\left[\frac{P \cdot q}{q^{2}+M^{2}}-\frac{P \cdot(q-P)}{(q-P)^{2}+M^{2}}\right],
\end{aligned}
$$

which must be satisfied even after regularization, therefore imposing

$$
\begin{aligned}
M & =\frac{16 M}{3 m_{G}^{2}} \int_{q} \frac{1}{q^{2}+M^{2}} \\
0 & \left.=\int_{q} \frac{q^{2}}{\left(q^{2}+M^{2}\right.}\right)^{2}
\end{aligned}
$$

Notice that Eq. (24) is merely the chiral-limit gap equation, whereas Eq. (25) entails that the axial-vector WGTI is satisfied if, and only if, the model is regularized so as to ensure there are no quadratic or logarithmic divergences [39].

\section{B. Contact interaction in modified RL}

Let us now consider the BS equation in the MRL truncation, Eq. (12). Supplemented by the effective gluon in the CI model, Eq. (14), and setting $\tilde{D}=4 / 3 m_{G}^{2}$, the modified homogeneous $\mathrm{BS}$ equation becomes

$$
\Gamma_{H}(P)=-\frac{4}{3 m_{G}^{2}} \int_{q}\left[\gamma_{\mu} \chi_{H}(P) \gamma_{\mu}-\xi \tilde{\Gamma}_{j} \chi_{H}(P) \tilde{\Gamma}_{j}\right],
$$

where we have made evident the momentum-independent nature of the BS amplitude and quark mass function. Recalling that $\tilde{\Gamma}_{j}=\left\{\mathbb{I}, \gamma_{5}, i / \sqrt{6} \sigma_{\mu \nu}\right\}$ and performing
Fierz transformatioon, it can be shown that the NL term, the one proportional to $\xi$, can be rewritten as $\frac{1}{3} \sigma_{\alpha \beta} \operatorname{tr}_{D}\left[\sigma_{\alpha \beta} \chi_{H}(P)\right]$ and does not contribute in the case of pseudoscalar and axial-vector mesons; it impacts, however, the vector meson case. Particularly, the $F_{1^{-}}(P)$ BS amplitude in Eq. (22) is no longer zero. This is a crucial difference with respect to the well known CI-RL truncation, which is clearly recovered in the limit case $\xi=0$.

The BS equation can be recast into an eigenvalue equation by finding proper projectors that decouple $E_{1^{-}}$and $F_{1^{-}}$in Eq. (22), such that Eq. (26) yields

$$
\left[\begin{array}{c}
E_{1^{-}}(P) \\
F_{1^{-}}(P)
\end{array}\right]=\frac{1}{3 \pi^{2} m_{G}^{2}}\left[\begin{array}{ll}
K_{E E}^{1^{-}} & K_{E F}^{1^{-}} \\
K_{F E}^{1^{-}} & K_{F F}^{1^{-}}
\end{array}\right]\left[\begin{array}{c}
E_{1^{-}}(P) \\
F_{1^{-}}(P)
\end{array}\right],
$$

where the integration kernels, $K_{i j}:=3 \pi^{2} m_{G}^{2} \mathcal{K}_{i j}$, are written as

$$
\begin{array}{r}
\mathcal{K}_{E E}^{1^{-}}=-P^{2} \tilde{I}\left(P^{2}\right), \quad \mathcal{K}_{E F}^{1^{-}}=-\frac{P^{2}}{2} I\left(P^{2}\right), \\
\mathcal{K}_{F E}^{1^{-}}=\eta_{\xi} I\left(P^{2}\right), \quad \mathcal{K}_{F F}^{1^{-}}=\eta_{\xi}\left(I\left(P^{2}\right)-\frac{P^{2}}{M^{2}} \tilde{I}\left(P^{2}\right)\right),
\end{array}
$$

the integrals:

$$
\begin{array}{r}
I\left(Q^{2}\right):=\frac{1}{3 \pi^{2} m_{G}^{2}} \int_{0}^{1} d \alpha \overline{\mathcal{C}}_{1}^{\mathrm{iu}}\left(\omega\left(M^{2}, \alpha, Q^{2}\right)\right), \\
\tilde{I}\left(Q^{2}\right):=\frac{1}{3 \pi^{2} m_{G}^{2}} \int_{0}^{1} d \alpha \alpha(1-\alpha) \overline{\mathcal{C}}_{1}^{\mathrm{iu}}\left(\omega\left(M^{2}, \alpha, Q^{2}\right),\right.
\end{array}
$$

the argument $\omega\left(M^{2}, \alpha, P^{2}\right):=M^{2}+\alpha(1-\alpha) P^{2} \quad$ and, finally,

$$
\eta_{\xi}:=\frac{2 M^{2} \xi}{3}, \quad \overline{\mathcal{C}}_{1}^{\mathrm{iu}}(\omega):=-\frac{d}{d \omega} \mathcal{C}^{\mathrm{iu}}(\omega) .
$$

Physical solutions of Eq. (27) are only valid for discrete values of $P^{2}=-m_{H}^{2}$. The smallest $P^{2}$ that satisfies the eigenvalue equation yields the ground-state meson mass. To produce other physical observables, the BS amplitudes must be canonically normalized, according to the condition

$$
1=2 N_{c} \frac{d}{d P^{2}}\left[\frac{1}{3} \operatorname{tr}_{D} \int_{q} \Gamma_{\mu}(-K) S(q) \Gamma_{\mu}(K) S(q-P)\right]_{K=P} .
$$

Computing the vector meson leptonic decay constant, and its tensor counter part, is straightforward from the expressions

$$
f_{v} m_{v}=\frac{N_{c}}{3} \int_{q} \operatorname{tr}_{D} \gamma_{\mu} S(q) \Gamma_{\mu} S(q-P),
$$


TABLE I. CI model results in the RL and MRL truncations. The model parameters: $m_{G}=0.132 \mathrm{GeV}, \Lambda_{\mathrm{UV}}=0.905 \mathrm{GeV}$, $\Lambda_{\mathrm{IR}}=0.240 \mathrm{GeV}$ and $\xi=0.6$. The current quark mass is set to $m=m_{u / d}=7 \mathrm{MeV}$, which yields $M=0.368 \mathrm{GeV}$.

\begin{tabular}{lllllllll}
\hline \hline$m_{\pi}$ & $f_{\pi}$ & $m_{a_{1}}$ & $m_{\rho}$ & $f_{\rho}$ & $f_{\rho}^{\perp}$ & $E_{\rho}$ & $F_{\rho}$ \\
\hline
\end{tabular}

\begin{tabular}{lllllllll}
\hline CI-RL & 0.140 & 0.101 & 1.08 & 0.929 & 0.129 & 0.133 & 1.531 & -
\end{tabular} $\begin{array}{lllllllll}\text { CI-MRL } & 0.140 & 0.101 & 1.08 & 0.770 & 0.125 & 0.134 & 1.230 & 0.503\end{array}$

$$
f_{v}^{\perp} m_{v}^{2}=\frac{N_{c}}{3} \int_{q} \sigma_{\mu \nu} P_{\nu} S(q) \Gamma_{\mu} S(q-P) .
$$

The computed masses and decay constants of $\pi$ and $\rho$ mesons, as well as the mass of the axial-vector $a_{1}$ meson, are collected in Table I, in both RL and MRL truncations. The parameter $\xi=0.6$ has been tuned to produce $m_{\rho}=$ $0.770 \mathrm{GeV}$, as compared with $m_{\rho}=0.929 \mathrm{GeV}$, obtained in the CI-RL; on the other hand, the decay constants barely change with varying $\xi$. Canonically normalized BS amplitudes of the $\rho$ meson are listed in Table I as well. To compute $\pi$ and $a_{1}$ listed properties we have followed standard literature, e.g., $[39,43,51]$

Since the NL term in the BS kernel does not contribute in the pseudoscalar and axial-vector channels, the static properties of $\pi$ and $a_{1}$ mesons remain unchanged. Subsequently, the mass of the $a_{1}$ meson is independent of $\xi$, thus fixed by its CI-RL value $m_{a_{1}}=1.08 \mathrm{GeV}$. Under such circumstances, the $a_{1}-\rho$ mass splitting is completely determined by the computed value of $m_{\rho}$, such that $m_{a_{1}}-$ $m_{\rho}=0.151 \mathrm{GeV}$ in the CI-RL truncation and $m_{a_{1}}-m_{\rho}=$ $0.310 \mathrm{GeV}$ in the CI-MRL case. Experimentally, one would expect $m_{a_{1}}-m_{\rho}=0.455 \mathrm{GeV}$ [52]. The latter might be obtained by doubling our preferred value of $\xi \rightarrow 2 \xi=1.2$, in such a way that $m_{\rho}=0.625 \mathrm{GeV}$, which is at some extent undesirable. One should rather focus on the failure of the RL and MRL truncations to describe the $a_{1}$ meson. The dressed quark ACM, enhanced by DCSB, produces a increased spin-orbit $(\mathrm{SO})$ repulsion in the case of $\ell=1$ mesons [16,24,26]. In neither case, RL nor MRL, the ACM term has been incorporated; as a consequence, $m_{a_{1}}$ is visibly underestimated. Often in CI model literature $[40,43,53]$, SO repulsion is mimicked by attaching a multiplicative factor $g_{\text {so }}$ to the RL kernel in the corresponding channels, tuned as $g_{\text {so }}=0.24$ to produce the empirical $a_{1}-\rho$ mass splitting. With the $\rho$ meson mass computed in the CI-MRL truncation, $g_{\text {so }} \approx 1 / 2$ yields the value $m_{a_{1}}-$ $m_{\rho} \approx 0.45 \mathrm{GeV}$. A more rigorous treatment of the $a_{1}$ meson is under investigation.

\section{QUARK-PHOTON VERTEX IN MODIFIED RL}

The quark-photon vertex in the CI-MRL satisfies the inhomogeneous BS equation

$$
\begin{aligned}
\Gamma_{\mu}^{\gamma}(Q)= & \gamma_{\mu}-\frac{4}{3 m_{G}^{2}} \int_{q} \gamma_{\alpha} S(q) \Gamma_{\mu}(Q) S(q-Q) \gamma_{\alpha} \\
& +\frac{4 \xi}{3 m_{G}^{2}} \int_{q} \tilde{\Gamma}_{j} S(q) \Gamma_{\mu}(Q) S(q-Q) \tilde{\Gamma}_{j}
\end{aligned}
$$

A general solution of Eq. (35) admits a decomposition in terms of 3 tensor structures, namely:

$$
\begin{aligned}
\Gamma_{\mu}^{\gamma}(Q) & =V_{1}\left(Q^{2}\right) \gamma_{\mu}^{L}+V_{2}\left(Q^{2}\right) \gamma_{\mu}^{T}+V_{3}\left(Q^{2}\right) \gamma_{\mu}^{A}, \\
\gamma_{\mu}^{L} & =\gamma_{\mu}-\gamma_{\mu}^{T}, \quad \gamma_{\mu}^{T}=\gamma_{\mu}-\frac{Q Q_{\mu}}{Q^{2}}, \quad \gamma_{\mu}^{A}=\frac{\sigma_{\mu \nu} Q_{\nu}}{M} .
\end{aligned}
$$

This simplicity is due to the momentum independent nature of the CI model. By solving Eq. (35), plainly one obtains that the longitudinal piece is simply

$$
V_{1}\left(Q^{2}\right):=P_{L}\left(Q^{2}\right)=1
$$

while the transverse dressing functions are expressed as

$$
\begin{gathered}
V_{2}\left(Q^{2}\right)=\left[P_{T}^{-1}\left(Q^{2}\right)+\eta_{\xi} \frac{Q^{2}}{2} \frac{I^{2}\left(Q^{2}\right)}{1-\eta_{\xi} \bar{I}\left(Q^{2}\right)}\right]^{-1}, \\
V_{3}\left(Q^{2}\right)=\frac{\eta_{\xi} I\left(Q^{2}\right)}{P_{T}^{-1}\left(Q^{2}\right)\left[1-\eta_{\xi} \bar{I}\left(Q^{2}\right)\right]+\eta_{\xi} \frac{Q^{2}}{2} I^{2}\left(Q^{2}\right)},
\end{gathered}
$$

where $P_{T}\left(Q^{2}\right):=\left[1+Q^{2} \tilde{I}\left(Q^{2}\right)\right]^{-1}$, and

$$
\bar{I}\left(Q^{2}\right):=I\left(Q^{2}\right)-\frac{Q^{2}}{M^{2}} \tilde{I}\left(Q^{2}\right) .
$$

The $V_{2}\left(Q^{2}\right)$ dressing function produces a timelike vector meson pole at $Q^{2}=-m_{\rho}^{2}$, while $V_{3}\left(Q^{2}\right)$ can be regarded as a profile function for the anomalous magnetic moment term, $\gamma_{\mu}^{A}$. Notably, in the $Q^{2} \rightarrow 0$ limit, the fully dressed QPV becomes

$$
\Gamma_{\mu}^{\gamma}(Q) \stackrel{Q^{2} \rightarrow 0}{=} \gamma_{\mu}+\frac{\sigma_{\mu \nu} Q_{\nu}}{2 M}\left(\frac{2 \eta_{\xi} I(0)}{1-\eta_{\xi} I(0)}\right),
$$

such that, with the parameters listed in Table I, one gets

$$
\zeta_{\mathrm{MRL}}:=\left(\frac{2 \eta_{\xi} I(0)}{1-\eta_{\xi} I(0)}\right) \approx 0.19
$$

The automatic incorporation of the AMM piece to the QPV, via inhomogeneous BS equation, is a desirable feature of the MRL truncation. The CI model plainly exposes it. This characteristic is not present in the RL approximation [26], and so in the CI-RL case; the latter corresponding to the case $\xi=0$, which implies $V_{2}\left(Q^{2}\right)=$ $P_{T}\left(Q^{2}\right)$ and $V_{3}\left(Q^{2}\right)=0$ [39,51]. Within the CI-RL 
approach, the AMM term is typically added by hand, such that the behavior of $V_{3}\left(Q^{2}\right)$ is modeled according to the exponential Ansatz [28-30]:

$$
V_{3}^{\mathrm{CI}-\mathrm{RL}}\left(Q^{2}\right):=\frac{\zeta_{\mathrm{CI}-\mathrm{RL}}}{2} \exp \left[-Q^{2} /\left(4 M^{2}\right)\right]
$$

where $\zeta_{\mathrm{RL}} \in[0,0.5]$ is a strength parameter, fully compatible with $\zeta_{\text {MRL }} \approx 0.19$. Thus, when dealing with the CI-RL truncation, we modify the QPV to account for the AMM, such that

$$
\Gamma_{\mu}^{\gamma} \stackrel{\mathrm{RL}}{:=} \gamma_{\mu}^{L}+\gamma_{\mu}^{T} P_{T}\left(Q^{2}\right)+\gamma_{A} V_{3}^{\mathrm{CI}-\mathrm{RL}}\left(Q^{2}\right)
$$

The vertex dressing $V_{2}\left(Q^{2}\right)$ is compared with its CI-RL counterpart, $P_{T}\left(Q^{2}\right)$, in the upper panel of Fig. 1. The presence of the vector meson pole at $Q^{2}=-m_{\rho}^{2}$ is a natural
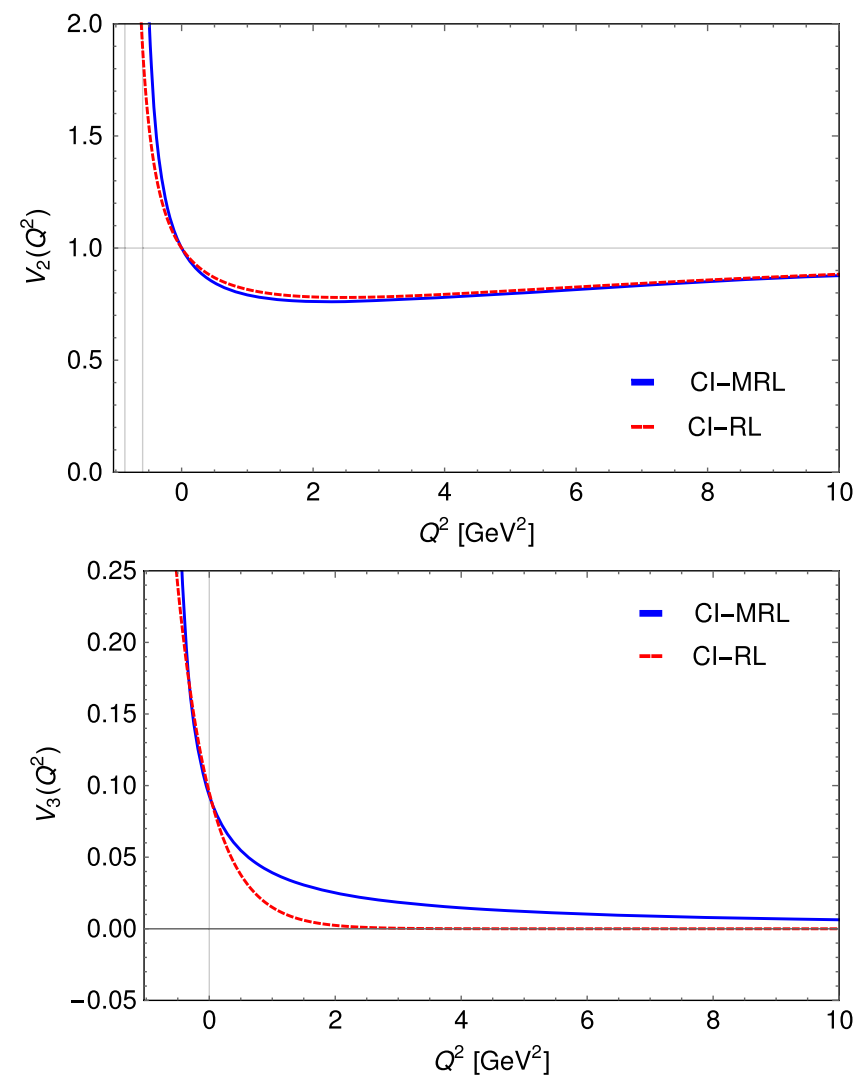

FIG. 1. Quark-photon vertex dressing functions as obtained from Eqs. (36)-(39). The upper panel displays the dressing function $V_{2}$, associated with the $\rho$ meson pole. Vertical grid lines indicate the location of the poles, $Q^{2}=-m_{\rho}^{2}$, such that $m_{\rho}=$ $0.929 \mathrm{GeV}$ in CI-RL and $m_{\rho}=0.770 \mathrm{GeV}$ in CI-MRL. Notably, $V_{2}\left(Q^{2}\right)=P_{T}\left(Q^{2}\right)$ in the CI-RL case. The lower panel depicts the dressing of the AMM piece of the QPV. Strictly speaking, the CI-RL case implies $V_{3}\left(Q^{2}\right)=0$; therefore, the Gaussian Ansatz from Eq. (43) and Refs. [28-30] is displayed instead $\left(\zeta_{\mathrm{CI}-\mathrm{RL}}=\zeta_{\mathrm{CI}-\mathrm{MRL}}=0.19\right)$. artifact of the RL truncation when obtaining the QPV through its corresponding BS equation [54-57]. It is then expected that electromagnetic form factors, in the vicinity of $Q^{2}=0$, be affected by the $\rho$ pole (for instance, it influences the associated charge radius) [58]; but the effects should be otherwise immaterial at large spacelike momenta $[13,58]$. The $Q^{2}$ profiles of the AMM dressing functions, in both truncations, are displayed in the lower panel of Fig. 1. It is clear that $V_{3}\left(Q^{2}\right)$ enhances the strength of the QPV in the low $Q^{2}$ domain, and its contribution vanishes as $Q^{2}$ grows; the CI-MRL case is power-law suppressed, in contrast with the Gaussian Ansatz in Eq. (43). In any case, the enhancement-damping patterns manifest in electromagnetic form factors involving spin- 1 mesons, but are strictly ruled out in elastic form factors of spin-0 mesons [28-30].

Finally, it is worth noticing that the QPV defined through Eqs. (36), (39) exhibits the correct asymptotic limit, i.e., $\Gamma_{\mu}^{\gamma}(Q) \rightarrow \gamma_{\mu}$ as $Q^{2} \rightarrow \infty$; therefore exposing that a dressed quark becomes pointlike for a large- $Q^{2}$ probe [59]. In the next section, we study the $\rho$ meson elastic EFF, capitalizing on how it is affected by the anomalous magnetic moment.

\section{ELECTROMAGNETIC FORM FACTORS}

The coupling of a photon to a $1^{-}$meson is characterized by three elastic form factors, such that the $\gamma \rho$ vertex can be expressed as [51]:

$$
\begin{aligned}
& \Lambda_{\lambda, \mu \nu}(K, Q)=\sum_{j=1}^{3} T_{\lambda, \mu \nu}^{(j)}(K, Q) F_{j}\left(Q^{2}\right) \\
& T_{\lambda, \mu \nu}^{(1)}(K, Q)= 2 K_{\lambda} P_{\mu \alpha}^{T}\left(p_{i}\right) P_{\alpha \nu}^{T}\left(p_{f}\right), \\
& T_{\lambda, \mu \nu}^{(2)}(K, Q)= {\left[Q_{\mu}-p_{\mu}^{i} \frac{Q^{2}}{2 m_{\rho}^{2}}\right] P_{\lambda \nu}^{T}\left(p_{f}\right) } \\
&-\left[Q_{\nu}+p_{\nu}^{f} \frac{Q^{2}}{2 m_{\rho}^{2}}\right] P_{\lambda \mu}^{T}\left(p_{i}\right), \\
& T_{\lambda, \mu \nu}^{(3)}(K, Q)=\frac{K_{\lambda}}{m_{\rho}^{2}}\left[Q_{\mu}-p_{\mu}^{i} \frac{Q^{2}}{2 m_{\rho}^{2}}\right]\left[Q_{\nu}+p_{\nu}^{f} \frac{Q^{2}}{2 m_{\rho}^{2}}\right] .
\end{aligned}
$$

The kinematic variables are defined as follows: $p^{i}=K-$ $Q / 2$ and $p^{f}=K+Q / 2$ denote the incoming and outgoing meson momenta, respectively, and $Q$ is the photon momentum; the on-shell conditions, $p_{i}^{2}=p_{f}^{2}=-m_{\rho}^{2}$, impose $K \cdot Q=0, K^{2}=-m_{\rho}^{2}-Q^{2} / 4$; and, as explained elsewhere [51], a symmetry-preserving treatment demands the WGTIs:

$$
p_{\mu}^{i} \Lambda_{\lambda, \mu \nu}=p_{\nu}^{f} \Lambda_{\lambda, \mu \nu}=Q_{\lambda} \Lambda_{\lambda, \mu \nu}=0 .
$$

In the impulse approximation [60], meson EFFs are completely described in terms of quark propagators, BS 
amplitudes and the QPV. Particularly, the elastic form factor of the $\rho$ meson reads

$$
\begin{aligned}
\Lambda_{\lambda, \mu \nu}\left(Q^{2}\right)= & 2 N_{c} \operatorname{tr} \int_{q}\left[\Gamma_{\nu}^{\rho}\left(-p_{f}\right) S\left(q+p_{f}\right)\right. \\
& \left.\times i \Gamma_{\lambda}^{\gamma}(Q) S\left(q+p_{i}\right) \Gamma_{\mu}^{\rho}\left(p_{i}\right) S(q)\right],
\end{aligned}
$$

where $\Gamma_{\mu}^{\rho}$ is the BS amplitude and $\Gamma_{\lambda}^{\gamma}(Q)$ is the QPV. Form factors are obtained from Eqs. (45), (46), (48), choosing appropriate projectors that decouple them from $\Lambda_{\lambda, \mu \nu}$. The particular expressions for $F_{i}\left(Q^{2}\right)$ are shown explicitly in Appendix A. It turns out convenient to relate the form factors $F_{j}\left(Q^{2}\right)$ with the electric, magnetic and quadrupole form factors, respectively

$$
\begin{aligned}
G_{E}\left(Q^{2}\right) & =F_{1}\left(Q^{2}\right)+\frac{2}{3} \frac{Q^{2}}{4 M_{\rho}^{2}} G_{Q}\left(Q^{2}\right), \\
G_{M}\left(Q^{2}\right) & =-F_{2}\left(Q^{2}\right), \\
G_{Q}\left(Q^{2}\right) & =F_{1}\left(Q^{2}\right)+F_{2}\left(Q^{2}\right)+F_{3}\left(Q^{2}\right)\left[1+\frac{Q^{2}}{4 M_{\rho}^{2}}\right] .
\end{aligned}
$$

Naturally, the $Q^{2} \rightarrow 0$ limit defines the charge, magnetic and quadrupole moments:

$$
\begin{aligned}
G_{E}\left(Q^{2} \rightarrow 0\right) & =1, \\
G_{M}\left(Q^{2} \rightarrow 0\right) & =\mu_{\rho}, \\
G_{Q}\left(Q^{2} \rightarrow 0\right) & =\mathcal{Q}_{\rho} .
\end{aligned}
$$

The computed form factors are depicted in Fig. 2. The electric form factor $G_{E}$ exhibits a zero and remains negative thereafter. For the CI-MRL truncation, the zero is located at

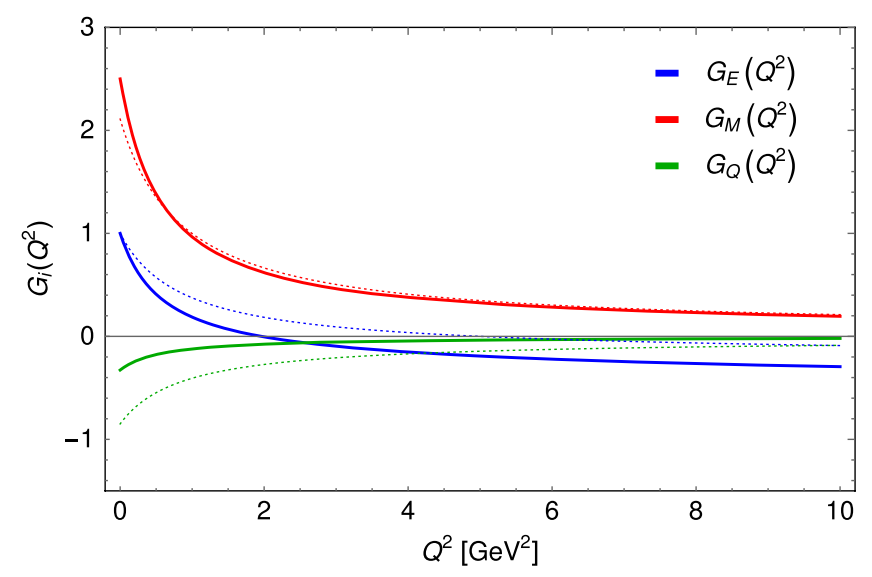

FIG. 2. $\rho$ meson form factors. From top to bottom: $G_{M}, G_{E}, G_{Q}$. Solid thick lines are the results obtained in the CI-MRL truncation, whereas the dotted lines are their analogous in the CI-RL approach [51].

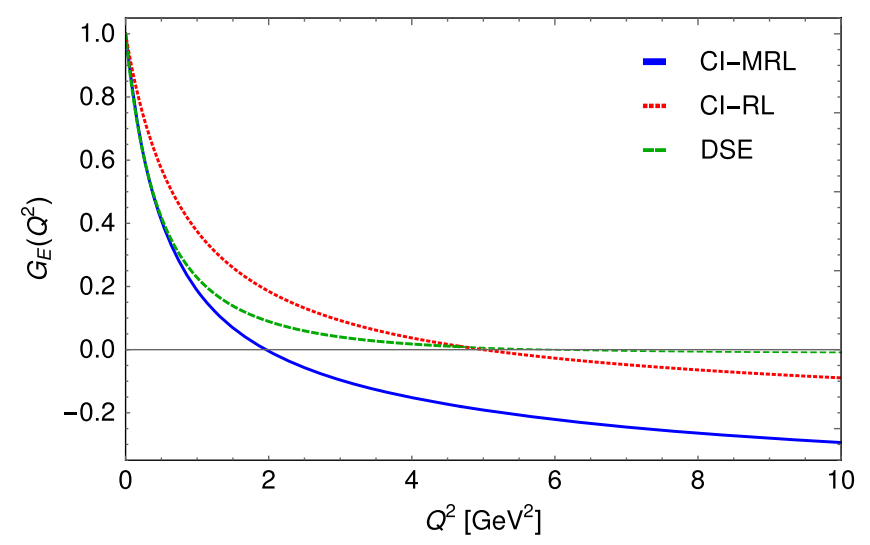

FIG. 3. $\rho$ meson electric form factor. Solid thick line corresponds to the CI-MRL result, whereas the dotted line depicts the CI-RL computation. We have included the DSE prediction from Ref. [61], which employs a RL approximation with a sophisticated gluon model. The large $Q^{2}$ behavior of the CI results is visibly harder.

$Q^{2} \approx 1.96 \mathrm{GeV}^{2}$, while the CI-RL exhibits a zero at $Q^{2} \approx 5 \mathrm{GeV}^{2}$. This crossing was predicted by the DSEBS approach from Ref. [61], although at $Q^{2} \approx 6 \mathrm{GeV}^{2}$. Figure 3 compares all of those calculations. Notably, although showing a steep drop toward its zero crossing, the CI-MRL result is in keen agreement with that produced in Ref. [61] at small momentum; the CI-RL shows a slower fall due to the larger value of $m_{\rho}$. As $Q^{2}$ increases, CI form factors are evidently harder, as a consequence of the limitations of the CI model (momentum independent mass function and BS amplitudes). The magnetic form factor $G_{M}$, also displayed in Fig. 4, turns out to be positive definite and monotonically decreasing, and quite similar in both truncations on the domain shown. The largest difference is appreciated at low $Q^{2}$. This can be attributed to the

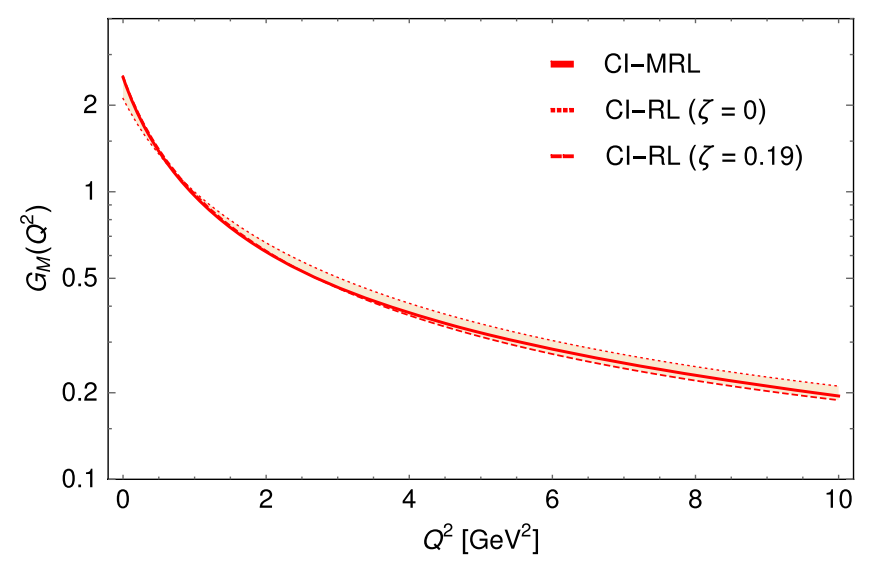

FIG. 4. $\rho$ meson magnetic form factor. Solid thick line corresponds to the CI-MRL result. For the CI-RL case, we have employed the QPV Ansatz from Eqs. (43), (44). With the strength parameter set to $\zeta_{\mathrm{CI}-\mathrm{RL}}=\zeta_{\mathrm{CI}-\mathrm{MRL}}=0.19$ in such case, the resemblance to the CI-MRL result is striking. 
TABLE II. $\rho$ meson static properties, as obtained from its elastic electromagnetic form factors. The CI-RL results match those from Ref. [51]. In our current framework, it corresponds to the $\xi=0$ limit of the CI-MRL truncation. For comparison, we have included novel DSE results from [61]. Additionally, Lattice QCD [62] predicts $\mu_{\rho}=2.21(8)$ and $r_{E}=0.82(4) \mathrm{fm}$, and the structureless meson limit dictates $\mu_{\rho}=2$ and $\mathcal{Q}_{\rho}=-1$.

\begin{tabular}{lccc}
\hline \hline & CI-MRL & CI-RL & DSE \\
\hline$r_{E} / \mathrm{fm}$ & 0.692 & 0.561 & 0.72 \\
$r_{M} / \mathrm{fm}$ & 0.603 & 0.515 & 0.69 \\
$r_{\mathcal{Q}} / \mathrm{fm}$ & 0.612 & 0.512 & - \\
$r_{E} m_{\rho}$ & 2.706 & 2.644 & 2.76 \\
$r_{M} m_{\rho}$ & 2.359 & 2.430 & 2.63 \\
$r_{\mathcal{Q}} m_{\rho}$ & 2.394 & 2.414 & - \\
$\mu_{\rho}$ & 2.500 & 2.110 & 2.01 \\
$\mathcal{Q}$ & -0.327 & -0.850 & -0.36 \\
\hline \hline
\end{tabular}

lack of AMM term in the QPV for the CI-RL truncation, which enhances the value of $G_{M}$ in a vicinity of $Q^{2}=0$, without altering the large $Q^{2}$ behavior. In Fig. 4 we also observe that, if the AMM piece is included in the CI-RL description, by using the vertex Ansatz from Eqs. (43), (44), magnetic form factors become even more similar. The effects on $G_{E}$ and $G_{Q}$ are immaterial and not shown. The quadrupole form factor $G_{Q}$ is negative and decreases in magnitude as $Q^{2}$ increases; CI-MRL and CI-RL truncations produce results with notorious different magnitudes.

Some static properties that can be read from the form factors are collected in Table II, namely charge, magnetic and quadrupole moments. For completeness, we have also included the corresponding radii, as defined from

$$
\left\langle r_{i}^{2}\right\rangle=-\left.6 \frac{1}{G_{i}(0)} \frac{d G_{i}\left(Q^{2}\right)}{d Q^{2}}\right|_{Q^{2}=0} .
$$

As can be inferred from Table II, the MRL truncation produces results which are closer to those obtained from using sophisticated gluon models $[57,61]$ and lattice QCD [62], whereas the CI-RL gives results closer to the structureless meson limit. As explained before, the QPV Ansatz from Eqs. (43), (44) makes the CI-RL magnetic form factor $G_{M}\left(Q^{2}\right)$ more similar to the CI-MRL case, and therefore the static properties derived from there.

\section{DISTRIBUTION AMPLITUDES}

To scrutinize a little further into the structure of the $\rho$ meson, and the effects of BS kernel truncation, we now examine the so called valence-quark distribution amplitudes. Particularly, let us consider two leading-twist distributions amplitudes of the $\rho$ meson: $\phi_{\|}(x)$ and $\phi_{\perp}(x)$. Intuitively, those describe the light-front momentum carried by the quark in a longitudinally or transversely polarized $\rho$,
[63]. In terms of quark propagators and BS amplitudes, the distributions can be written as [64]:

$$
\begin{aligned}
n \cdot P f_{\rho} \phi_{\|}(x) & =m_{\rho} N_{c} \operatorname{tr} \int_{q} \delta_{n}^{x}\left(q^{+}\right) \gamma \cdot n n_{\nu} \chi_{\nu}^{\rho}(q ; P), \\
f_{\rho}^{\perp} \phi_{\perp}(x) & =-\frac{1}{2} N_{c} \operatorname{tr} \int_{q} \delta_{n}^{x}\left(q^{+}\right) n_{\mu} \sigma_{\mu \alpha} \mathcal{O}_{\alpha \nu}^{\perp} \chi_{\nu}^{\rho}(q ; P),
\end{aligned}
$$

where $\delta_{n}^{x}\left(q^{+}\right):=\delta(n \cdot q-x n \cdot P)$ and $\mathcal{O}_{\alpha \nu}^{\perp}=\delta_{\alpha \nu}+n_{\alpha} \bar{n}_{\nu}+$ $\bar{n}_{\alpha} n_{\nu} ; n$ is a lightlike four-vector such that $n^{2}=0$, $n \cdot P=-m_{\rho} ; \bar{n}$ is a conjugate lightlike four-vector, $\bar{n}^{2}=0, n \cdot \bar{n}=-1$; and, with the above definitions, $\int_{0}^{1} d x \phi_{\|}(x)=\int_{0}^{1} d x \phi_{\perp}(x)=1$.

The PDAs can be derived from their corresponding Mellin moments, $\left\langle x^{m}\right\rangle=\int_{0}^{1} d x x^{m} \phi(x)$. After evaluating the Dirac trace, a series of algebraic manipulations involving Feynman parametrization, supplemented by the uniqueness of Mellin moments, enable us to identify

$$
\begin{gathered}
\phi_{\|}(x)=\frac{1}{f_{\rho}} \frac{N_{c}}{4 \pi^{2}} m_{\rho}\left[2 x(1-x) E_{1^{-}}+F_{1^{-}}\right] \\
\times \overline{\mathcal{C}}_{1}^{\mathrm{iu}}\left(\omega\left(M^{2}, x,-m_{\rho}^{2}\right)\right), \\
\phi_{\perp}(x)=\frac{1}{f_{\rho}^{\perp}} \frac{N_{c}}{4 \pi^{2}} M\left[E_{1^{-}}+\left(1+\frac{m_{1^{-}}^{2}}{M^{2}} x(1-x)\right) F_{1^{-}}\right] \\
\times \overline{\mathcal{C}}_{1}^{\mathrm{iu}}\left(\omega\left(M^{2}, x,-m_{\rho}^{2}\right)\right) .
\end{gathered}
$$

The obtained PDAs are displayed in Fig. 5. Both distributions manifest symmetry under the exchange $1 \leftrightarrow 1-x$, which is expected in the isospin symmetric limit $m_{u}=m_{d}$. The parallel distribution, $\phi_{\|}$, is more compressed with respect to its perpendicular counterpart, an expect pattern from sophisticated momentum-dependent interactions [64]. It is evident that the derived PDAs do not

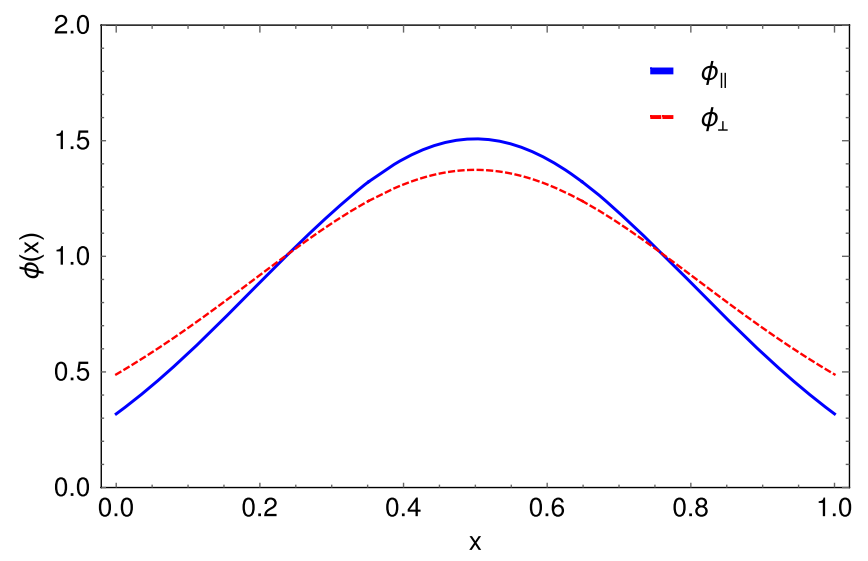

FIG. 5. $\rho$ meson PDAs. Computed distributions, Eq. (54), in the CI-MRL truncation. 

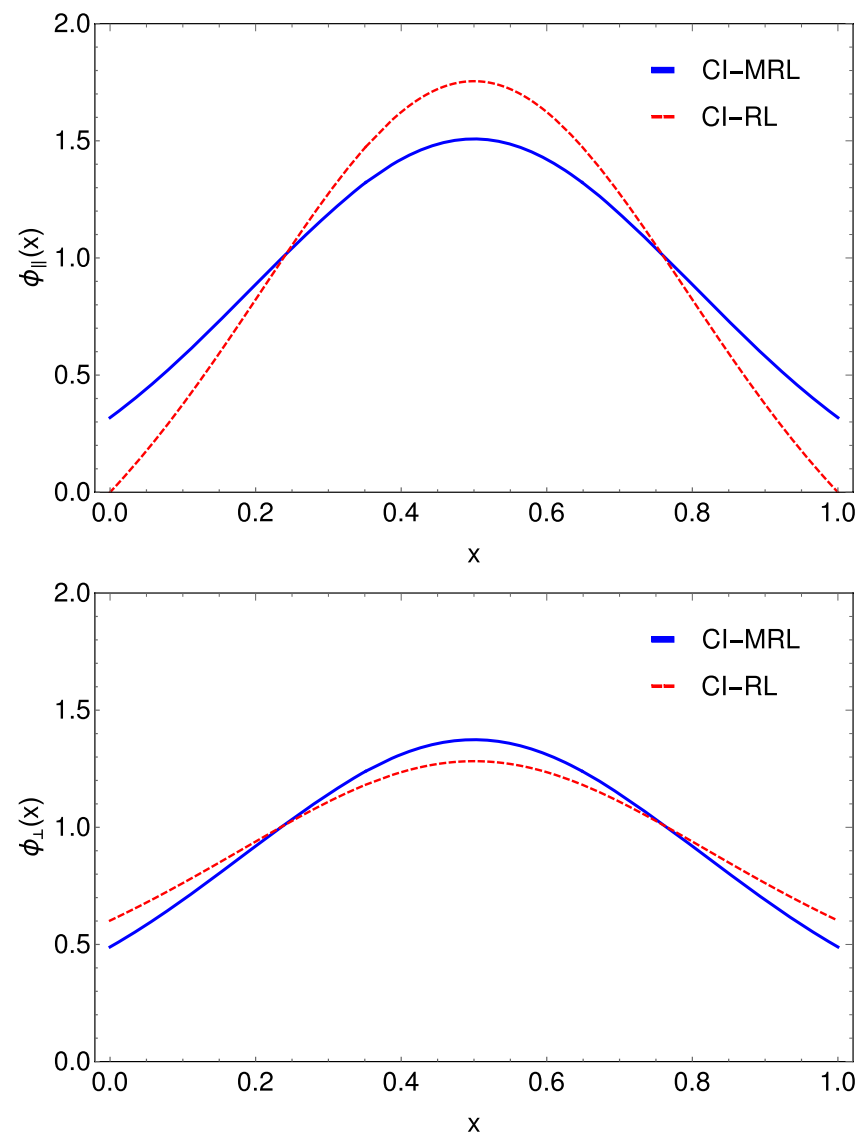

FIG. 6. $\rho$ meson PDAs. Computed distributions, Eq. (54), in the CI-RL and CI-MRL truncations. Notably, $\phi_{\|}(x)$ in the CI-RL exhibits soft endpoint behavior.

vanish at the endpoints, in contradiction with QCD prescriptions [63-65]. The same occurs for the pion leadingtwist PDA in the CI model, for which $\phi_{\pi}(x)=1$ in the chiral limit [39]. Nonetheless, it is quite interesting that, in the absence of the $F_{\rho}$ BS amplitude (the CI-RL case), the distribution $\phi_{\|}$actually exhibits soft endpoint behavior. This is in apparent contradiction with the observed patterns, which is also well understood from Eqs. (54) and drawn in Fig. 6.

\section{CONCLUSIONS}

Starting from the leading-order symmetry-preserving RL truncation for the two-body problem, we have illustrated how, although the vector and axial-vector WGTIs provide necessary consistency conditions to construct the interrelated one body and two body kernels, these turn out insufficient to determine the kernels in an unambigous manner [25]. Particularly, we construct a two-body kernel completely consistent with the aforementioned WGTIs that includes NL terms. The MRL truncation lets unaltered the pseudoscalar and axial-vector meson properties, but yields some implications in the vector channel. This can be well illustrated by employing the CI model described in Sec. III. Among other things, we have seen how solutions of the QPV BS equations, using the MRL kernel, automatically generate an anomalous magnetic moment term in the vertex structure. This is in addition to the vector meson pole at $Q^{2}=-m_{\rho}^{2}$, which is an artifact of the RL truncation [56]. In the presence of DCSB, a dressed light-quark possesses a large anomalous electromagnetic moment [26,27]. Therefore, the automatic incorporation of such term is an important outcome of the constructed two-body kernel in MRL truncation. Immediate consequences are naturally noted in the $\rho$ meson properties. For instance, although the produced decay constants are practically invariant under the effects of the NL pieces we have introduced in the BS kernel, the experimental mass of the $\rho$ can be faithfully obtained within the CI-MRL, in contrast with the CI-RL approach which produces a larger value [51]. In some way, the NL term of the two-body kernel mimics the effects of the pion cloud effects, which correct the inflated masses produced when they are not considered [66]; whether or not our kernel is actually related to pion cloud effects is currently under investigation. On another note, whereas the CI-RL truncation yields static properties (as derived from the corresponding EFF) closer to the structureless meson limit, CI-MRL results are more compatible with those obtained from more sophisticated approaches $[61,62]$. Focusing on the magnetic form factor, we have reinforced the importance of the AMM for a good description of vector mesons. Finally, we have derived the $\rho$ meson $\phi_{\|}(x)$ and $\phi_{\perp}(x)$ distribution amplitudes. The necessity of employing a regularization scheme, and subsequently cutoffs, to deal with the CI model, makes the PDAs not vanishing at the endpoints. This is also observed in the pion case: the chiral limit pseudoscalar yields $\phi_{\pi}(x)=1$ [39]. Interestingly, in the absence of the $F_{\rho}$ piece of the BetheSalpeter amplitude (the CI-RL case), the distribution $\phi_{\|}(x)$ actually vanishes at the endpoints. Although it is an expected behavior from QCD grounds, [64], it seems rather incompatible with the pion result and the CI framework in general. Having exposed some impacts of the two-body kernel on the QPV structure and $\rho$ meson properties, via masses and decay constants, electromagnetic form factors and distribution amplitudes, the question on its implications on the distribution functions remains unanswered. This is an aspect of the $\rho$ meson structural properties that will be addressed elsewhere. A similar study for the open flavor case, in particular the Kaon sector, will be conducted as well.

\section{ACKNOWLEDGMENTS}

The authors acknowledge valuable comments from José Rodríguez-Quintero. This work was supported by National Natural Science Foundation of China under Grant No. 12135007. 


\section{APPENDIX: APPENDIX $\rho$ FORM FACTORS}

The vertex characterizing the elastic EFFs of the $\rho$ meson is written in Eq. (46). With properly chosen projection operators, the three form factors $F_{i}\left(Q^{2}\right)$ are written, in general:

$$
F_{i}=\int_{0}^{1} d u_{1} \int_{0}^{1-u 1} d u_{2} \mathcal{A}_{i} \overline{\mathcal{C}}_{1}^{\mathrm{iu}}(\Omega)+\mathcal{B}_{i} \overline{\mathcal{C}}_{2}^{\mathrm{iu}}(\Omega)
$$

where $\Omega\left(u_{1}, u_{2}, M^{2}, P^{2}, Q^{2}\right)=M^{2}-P^{2}\left(u_{1}+u_{2}\right)\left(u_{1}+u_{2}-1\right)+u_{1} u_{2} Q^{2}$ and $\overline{\mathcal{C}}_{2}^{\text {iu }}(\Omega)=\frac{d^{2}}{d \Omega^{2}} \mathcal{C}^{\text {iu }}(\Omega) . \mathcal{X}_{i}=\mathcal{X}_{i}^{E E}\left(Q^{2}\right) E_{c}^{2}+$ $\mathcal{X}_{i}^{E F}\left(Q^{2}\right) E_{c} F_{c}+\mathcal{X}_{i}^{F F}\left(Q^{2}\right) F_{c}^{2}$, for $\mathcal{X}=\mathcal{A}, \mathcal{B}$ and $i=1,2$, 3. Explicitly, we arrive at the following expressions for the different pieces:

$$
\begin{gathered}
\mathcal{A}_{1}^{E E}\left(Q^{2}\right)=-\frac{N_{c}}{4 \pi^{2}}\left\{\frac{Q^{2}\left(u_{1}-u_{2}\right)\left(6 m_{\rho}^{4}+5 m_{\rho}^{2} Q^{2}+Q^{4}\right)}{4 m_{\rho}^{4}\left(Q^{2}+4 m_{\rho}^{2}\right)} V_{1}\left(Q^{2}\right)+\left(u_{1}+u_{2}-2\right) V_{2}\left(Q^{2}\right)\right\} \\
\mathcal{A}_{1}^{E F}\left(Q^{2}\right)=\frac{N_{c}}{4 \pi^{2}}\left\{4 V_{2}\left(Q^{2}\right)-\frac{Q^{2}\left(u_{1}+u_{2}\right)}{M^{2}} V_{3}\left(Q^{2}\right)\right\} \\
\mathcal{A}_{1}^{F F}\left(Q^{2}\right)=\frac{N_{c}}{4 \pi^{2}}\left\{\frac{Q^{2}\left(u_{1}-u_{2}\right)\left(Q^{2}+2 m_{\rho}^{2}\right)}{4 M^{2}\left(Q^{2}+4 m_{\rho}^{2}\right)} V_{1}\left(Q^{2}\right)+\frac{m_{\rho}^{2}}{M^{2}}\left(u_{1}+u_{2}\right) V_{2}\left(Q^{2}\right)\right\} \\
\mathcal{A}_{2}^{E E}\left(Q^{2}\right)=-\frac{N_{c}}{4 \pi^{2}}\left\{\frac{2\left(u_{1}-u_{2}\right)\left(Q^{2}+3 m_{\rho}^{2}\right)}{Q^{2}+4 m_{\rho}^{2}} V_{1}\left(Q^{2}\right)+2\left(u_{1}+1\right) V_{2}\left(Q^{2}\right)+4 V_{3}\left(Q^{2}\right)\right\} \\
\mathcal{A}_{2}^{E F}\left(Q^{2}\right)=\frac{N_{c}}{4 \pi^{2}}\left\{-4 V_{2}\left(Q^{2}\right)+\frac{4 m_{\rho}^{2}\left(u_{1}+u_{2}-1\right)+2 u_{2} Q^{2}}{M^{2}} V_{3}\left(Q^{2}\right)\right\} \\
\mathcal{A}_{2}^{F F}\left(Q^{2}\right)=\frac{N_{c}}{4 \pi^{2}}\left\{\frac{2 m_{\rho}^{4}\left(u_{1}-u_{2}\right)}{M^{2}\left(Q^{2}+4 m_{\rho}^{2}\right)} V_{1}\left(Q^{2}\right)-\frac{2 u_{2} m_{\rho}^{2}}{M^{2}} V_{2}\left(Q^{2}\right)\right\} \\
\mathcal{A}_{3}^{E E}\left(Q^{2}\right)=\frac{N_{c}}{4 \pi^{2}}\left\{\frac{\left(u_{1}-u_{2}\right)\left(60 m_{\rho}^{6}+32 m_{\rho}^{4} Q^{2}+7 m_{\rho}^{2} Q^{4}+Q^{6}\right)}{m_{\rho}^{2}\left(Q^{2}+4 m_{\rho}^{2}\right)^{2}} V_{1}\left(Q^{2}\right)+4 \frac{m_{\rho}^{2}\left(u_{1}-u_{2}\right)}{Q^{2}+4 m_{\rho}^{2}} V_{2}\left(Q^{2}\right)\right\} \\
\mathcal{A}_{3}^{E F}\left(Q^{2}\right)=\frac{N_{c}}{4 \pi^{2}}\left\{\frac{4 m_{\rho}^{2} Q^{2}\left(u_{1}-u_{2}\right)}{M^{2}\left(Q^{2}+4 m_{\rho}^{2}\right)} V_{3}\left(Q^{2}\right)\right\} \\
\mathcal{A}_{3}^{F F}\left(Q^{2}\right)=\frac{N_{c}}{4 \pi^{2}}\left\{-\frac{m_{\rho}^{2}\left(u_{1}-u_{2}\right)\left(20 m_{\rho}^{4}+4 m_{\rho}^{2} Q^{2}+Q^{4}\right)}{M^{2}\left(Q^{2}+4 m_{\rho}^{2}\right)^{2}} V_{1}\left(Q^{2}\right)+\frac{4 m_{\rho}^{4}\left(u_{2}-u_{1}\right)}{M^{2}\left(Q^{2}+4 m_{\rho}^{2}\right)} V_{2}\left(Q^{2}\right)\right\}
\end{gathered}
$$

$$
\begin{aligned}
& \mathcal{B}_{1}^{E E}\left(Q^{2}\right) \\
& =\frac{N_{c}}{4 \pi^{2}}\left\{\frac{Q^{2}\left(u_{1}-u_{2}\right)\left(Q^{2}+2 m_{\rho}^{2}\right)}{8 m_{\rho}^{4}\left(Q^{2}+4 m_{\rho}^{2}\right)}\right. \\
& \quad \times\left[-M^{2}\left(Q^{2}+3 m_{\rho}^{2}\right)+m_{\rho}^{4}\left(u_{1}+u_{2}-1\right)\left(9 u_{1}+9 u_{2}-2\right)+m_{\rho}^{2} Q^{2}\left(\left(7 u_{1}-3\right) u_{2}+3\left(u_{1}-1\right) u_{1}+3 u_{2}^{2}\right)+u_{1} u_{2} Q^{4}\right] V_{1}\left(Q^{2}\right) \\
& \left.\quad+\frac{1}{2}\left[M^{2}\left(2-u_{1}-u_{2}\right)+m_{\rho}^{2}\left(u_{1}+u_{2}-1\right)\left(u_{1}+u_{2}\right)\left(3 u_{1}+3 u_{2}-4\right)+3 u_{1} u_{2}\left(u_{1}+u_{2}-2\right) Q^{2}\right] V_{2}\left(Q^{2}\right)-Q^{2} V_{3}\left(Q^{2}\right)\right\} \\
& \mathcal{B}_{1}^{E F}\left(Q^{2}\right) \\
& =\frac{N_{c}}{4 \pi^{2}}\left\{-\frac{Q^{2}\left(u_{1}-u_{2}\right)\left(Q^{2}+2 m_{\rho}^{2}\right)}{4 m_{\rho}^{2}} V_{1}\left(Q^{2}\right)-\frac{8 m_{\rho}^{4}\left(u_{1}+u_{2}-1\right)\left(2 u_{1}+2 u_{2}+1\right)+4 m_{\rho}^{2} Q^{2}\left(4 u_{1} u_{2}+u_{1}+u_{2}\right)}{4 m_{\rho}^{2}} V_{2}\left(Q^{2}\right)\right. \\
& \left.\quad+\frac{Q^{2}\left(\left(u_{1}+u_{2}\right)\left(m_{\rho}^{2}\left(u_{1}+u_{2}-1\right)\left(3 u_{1}+3 u_{2}+2\right)+3 u_{1} u_{2} Q^{2}\right)-M^{2}\left(u_{1}+u_{2}+4\right)\right)}{2 M^{2}} V_{3}\left(Q^{2}\right)\right\}
\end{aligned}
$$




$$
\begin{aligned}
\mathcal{B}_{1}^{F F}\left(Q^{2}\right) \\
=\frac{N_{c}}{4 \pi^{2}}\left\{\frac{Q^{2}\left(u_{1}-u_{2}\right)\left(Q^{2}+2 m_{\rho}^{2}\right)\left(-3 M^{2}+m_{\rho}^{2}\left(u_{1}+u_{2}-1\right)\left(u_{1}+u_{2}+2\right)+u_{1} u_{2} Q^{2}\right)}{8 M^{2}\left(Q^{2}+4 m_{\rho}^{2}\right)} V_{1}\left(Q^{2}\right)\right. \\
+\left[\frac{m_{\rho}^{2}\left(m_{\rho}^{2}\left(u_{1}+u_{2}-2\right)\left(u_{1}+u_{2}-1\right)\left(u_{1}+u_{2}\right)+Q^{2}\left(-u_{1}^{2} u_{2}+2 u_{1}^{2}\left(u_{1}-1\right)-\left(2+u_{1}\right) u_{2}^{2}+2 u_{2}^{3}\right)\right)}{2 M^{2}}\right. \\
\left.-\frac{1}{2}\left(m_{\rho}^{2}\left(3 u_{1}+3 u_{2}-4\right)+2 Q^{2}\left(u_{1}+u_{2}\right)\right)\right] V_{2}\left(Q^{2}\right) \\
\left.+\frac{Q^{2}\left(-M^{2}+m_{\rho}^{2}\left(u_{1}+u_{2}-2\right)\left(u_{1}+u_{2}\right)+u_{1} u_{2} Q^{2}\right)}{M^{2}} V_{3}\left(Q^{2}\right)\right\}
\end{aligned}
$$

$$
\begin{aligned}
& \mathcal{B}_{2}^{E E}\left(Q^{2}\right) \\
& =\frac{N_{c}}{4 \pi^{2}}\left\{\frac{\left(u_{1}-u_{2}\right)}{Q^{2}+4 m_{\rho}^{2}}\right. \\
& \times\left[-M^{2}\left(Q^{2}+3 m_{\rho}^{2}\right)+m_{\rho}^{4}\left(u_{1}+u_{2}-1\right)\left(9 u_{1}+9 u_{2}-2\right)+m_{\rho}^{2} Q^{2}\left(\left(7 u_{1}-3\right) u_{2}+3\left(u_{1}-1\right) u_{1}+3 u_{2}^{2}\right)+u_{1} u_{2} Q^{4}\right] V_{1}\left(Q^{2}\right) \\
& +\left[-\left(u_{1}+1\right) M^{2}+m_{\rho}^{2}\left(u_{1}+u_{2}-1\right)\left(3 u_{1}^{2}+3 u_{1} u_{2}+u_{1}+3 u_{2}\right)+u_{1}\left(3 u_{1}+1\right) u_{2} Q^{2}\right] V_{2}\left(Q^{2}\right) \\
& \left.+\left[2 m_{\rho}^{2}\left(u_{1}+u_{2}-1\right)\left(2 u_{1}+2 u_{2}+1\right)+2 u_{1}\left(2 u_{2}+1\right) Q^{2}\right] V_{3}\left(Q^{2}\right)\right\} \\
& \mathcal{B}_{2}^{E F}\left(Q^{2}\right) \\
& =\frac{N_{c}}{4 \pi^{2}}\left\{\left[2 m_{\rho}^{2}\left(u_{2}-u_{1}\right)\right] V_{1}\left(Q^{2}\right)+\left[2\left(m_{\rho}^{2}\left(2 u_{1}^{2}+u_{1}\left(4 u_{2}-3\right)+u_{2}\left(2 u_{2}-3\right)-1\right)+2 u_{1} u_{2} Q^{2}\right)\right] V_{2}\left(Q^{2}\right)\right. \\
& +\left[\frac{-6 m_{\rho}^{4}\left(u_{1}+u_{2}-1\right)^{2}\left(u_{1}+u_{2}\right)+m_{\rho}^{2} Q^{2}\left(-2 u_{1}^{3}+u_{1}^{2}\left(2-5 u_{2}\right)+u_{1}\left(7-6 u_{2}\right) u_{2}-3\left(u_{2}-1\right) u_{2}^{2}\right)-u_{1} u_{2} Q^{4}}{M^{2}}\right. \\
& \left.\left.+2 m_{\rho}^{2}\left(u_{1}+u_{2}-3\right)+Q^{2}\left(2 u_{1}+u_{2}\right)\right] V_{3}\left(Q^{2}\right)\right\} \\
& \mathcal{B}_{2}^{F F}\left(Q^{2}\right) \\
& =\frac{N_{c}}{4 \pi^{2}}\left\{\frac{m_{\rho}^{4}\left(u_{1}-u_{2}\right)\left(-3 M^{2}+m_{\rho}^{2}\left(u_{1}+u_{2}-1\right)\left(u_{1}+u_{2}+2\right)+u_{1} u_{2} Q^{2}\right)}{M^{2}\left(Q^{2}+4 m_{\rho}^{2}\right)} V_{1}\left(Q^{2}\right)\right. \\
& +\left[\frac{m_{\rho}^{2}\left(-\left(u_{2}+2\right) M^{2}+m_{\rho}^{2}\left(u_{1}+u_{2}-1\right)\left(3 u_{1} u_{2}+2 u_{1}+3 u_{2}^{2}\right)+3 u_{1} u_{2}^{2} Q^{2}\right)}{M^{2}}\right] V_{2}\left(Q^{2}\right) \\
& \left.+\frac{2 m_{\rho}^{2}\left(-M^{2}+m_{\rho}^{2}\left(u_{1}+u_{2}-1\right)\left(u_{1}+u_{2}+1\right)+\left(u_{1}+1\right) u_{2} Q^{2}\right)}{M^{2}} V_{3}\left(Q^{2}\right)\right\} \\
& \mathcal{B}_{3}^{E E}\left(Q^{2}\right) \\
& =\frac{N_{c}}{4 \pi^{2}}\left\{\frac{-\left(u_{1}-u_{2}\right)\left(20 m_{\rho}^{4}+4 m_{\rho}^{2} Q^{2}+Q^{4}\right)}{2 m_{\rho}^{2}\left(Q^{2}+4 m_{\rho}^{2}\right)^{2}}\right. \\
& \times\left[-M^{2}\left(Q^{2}+3 m_{\rho}^{2}\right)+m_{\rho}^{4}\left(u_{1}+u_{2}-1\right)\left(9 u_{1}+9 u_{2}-2\right)+m_{\rho}^{2} Q^{2}\left(\left(7 u_{1}-3\right) u_{2}+3\left(u_{1}-1\right) u_{1}+3 u_{2}^{2}\right)+u_{1} u_{2} Q^{4}\right] V_{1}\left(Q^{2}\right) \\
& +\frac{2 m_{\rho}^{2}\left(M^{2}\left(u_{1}-u_{2}\right)-m_{\rho}^{2}\left(u_{1}+u_{2}-1\right)\left(2\left(8 u_{1}+1\right) u_{2}+u_{1}\left(3 u_{1}-2\right)-3 u_{2}^{2}\right)-u_{1} u_{2} Q^{2}\left(7 u_{1}+u_{2}-4\right)\right)}{Q^{2}+4 m_{\rho}^{2}} V_{2}\left(Q^{2}\right) \\
& \left.-\frac{4 m_{\rho}^{2}\left(4 m_{\rho}^{2}\left(u_{1}+u_{2}-1\right)+\left(2 u_{1}-1\right) Q^{2}\right)}{Q^{2}+4 m_{\rho}^{2}} V_{3}\left(Q^{2}\right)\right\}
\end{aligned}
$$




$$
\begin{aligned}
\mathcal{B}_{3}^{E F}\left(Q^{2}\right) \\
=\frac{N_{c}}{4 \pi^{2}}\left\{\frac{\left(u_{1}-u_{2}\right)\left(20 m_{\rho}^{4}+4 m_{\rho}^{2} Q^{2}+Q^{4}\right)}{Q^{2}+4 m_{\rho}^{2}} V_{1}\left(Q^{2}\right)+\left[4 m_{\rho}^{2}\left(u_{1}+u_{2}\right)\right] V_{2}\left(Q^{2}\right)\right. \\
\quad+\frac{2 m_{\rho}^{2}}{M^{2}\left(Q^{2}+4 m_{\rho}^{2}\right)}\left[-M^{2}\left(8 m_{\rho}^{2}\left(u_{1}+u_{2}-2\right)+Q^{2}\left(3 u_{1}+u_{2}-4\right)\right)+8 m_{\rho}^{4}\left(u_{1}+u_{2}-1\right)^{2}\left(u_{1}+u_{2}\right)\right. \\
\left.\left.\quad+m_{\rho}^{2} Q^{2}\left(u_{1}+u_{2}\right)\left(-4 u_{1} u_{2}+\left(u_{1}-3\right) u_{1}+3 u_{2}^{2}-5 u_{2}+2\right)-u_{1} u_{2} Q^{4}\left(3 u_{1}+u_{2}\right)\right] V_{3}\left(Q^{2}\right)\right\} \\
\mathcal{B}_{3}^{F F}\left(Q^{2}\right) \\
=\frac{N_{c}}{4 \pi^{2}}\left\{-\frac{m_{\rho}^{2}\left(u_{1}-u_{2}\right)\left(20 m_{\rho}^{4}+4 m_{\rho}^{2} Q^{2}+Q^{4}\right)\left(-3 M^{2}+m_{\rho}^{2}\left(u_{1}+u_{2}-1\right)\left(u_{1}+u_{2}+2\right)+u_{1} u_{2} Q^{2}\right)}{2 M^{2}\left(Q^{2}+4 m_{\rho}^{2}\right)^{2}} V_{1}\left(Q^{2}\right)\right. \\
\quad+\frac{2 m_{\rho}^{2}}{M^{2}\left(Q^{2}+4 m_{\rho}^{2}\right)}\left[M^{2}\left(m_{\rho}^{2}\left(7 u_{1}+9 u_{2}\right)+2 Q^{2}\left(u_{1}+u_{2}\right)\right)+m_{\rho}^{2}\left(Q^{2}\left(-2 u_{1}^{3}+u_{1}^{2}\left(u_{2}+2\right)-5 u_{1} u_{2}^{2}-2\left(u_{2}-1\right) u_{2}^{2}\right)\right.\right. \\
\left.\left.\quad-m_{\rho}^{2}\left(u_{1}+u_{2}-1\right)\left(u_{1}\left(5 u_{1}+2\right)+u_{2}\left(11 u_{2}-2\right)\right)\right)\right] V_{2}\left(Q^{2}\right) \\
\left.\quad-\frac{4 m_{\rho}^{2}\left(-M^{2}\left(Q^{2}+4 m_{\rho}^{2}\right)+4 m_{\rho}^{4}\left(u_{1}+u_{2}-1\right)\left(u_{1}+u_{2}\right)+m_{\rho}^{2} Q^{2}\left(6 u_{1} u_{2}+\left(u_{1}-2\right) u_{1}+u_{2}^{2}\right)+u_{1} u_{2} Q^{4}\right)}{M^{2}\left(Q^{2}+4 m_{\rho}^{2}\right)} V_{3}\left(Q^{2}\right)\right\}
\end{aligned}
$$

[1] C. D. Roberts, Symmetry 12, 1468 (2020).

[2] C. D. Roberts and A. G. Williams, Prog. Part. Nucl. Phys. 33, 477 (1994).

[3] C. S. Fischer, Prog. Part. Nucl. Phys. 105, 1 (2019).

[4] L. D. Faddeev, Sov. Phys. Dokl. 6, 384 (1961).

[5] E. E. Salpeter and H. A. Bethe, Phys. Rev. 84, 1232 (1951).

[6] G. Eichmann, H. Sanchis-Alepuz, R. Williams, R. Alkofer, and C. S. Fischer, Prog. Part. Nucl. Phys. 91, 1 (2016).

[7] Z.-F. Cui, M. Ding, F. Gao, K. Raya, D. Binosi, L. Chang, C. D. Roberts, J. Rodríguez-Quintero, and S. M. Schmidt, Eur. Phys. J. C 80, 1064 (2020).

[8] J. Arrington et al., J. Phys. G 48, 075106 (2021).

[9] S.-X. Qin, C. D. Roberts, and S. M. Schmidt, Few Body Syst. 60, 26 (2019).

[10] T. Hilger, C. Popovici, M. Gomez-Rocha, and A. Krassnigg, Phys. Rev. D 91, 034013 (2015).

[11] K. Raya, A. Bashir, and P. Roig, Phys. Rev. D 101, 074021 (2020).

[12] M. Chen, L. Chang, and Y.-X. Liu, Phys. Rev. D 101, 056002 (2020).

[13] K. Raya, L. Chang, A. Bashir, J. J. Cobos-Martinez, L. X. Gutiérrez-Guerrero, C. D. Roberts, and P. C. Tandy, Phys. Rev. D 93, 074017 (2016).

[14] L. Chang, I. C. Cloët, C. D. Roberts, S. M. Schmidt, and P. C. Tandy, Phys. Rev. Lett. 111, 141802 (2013).

[15] C. Chen, C. S. Fischer, C. D. Roberts, and J. Segovia, Phys. Lett. B 815, 136150 (2021).
[16] S.-X. Qin and C. D. Roberts, Chin. Phys. Lett. 37, 121201 (2020).

[17] D. Binosi, L. Chang, J. Papavassiliou, S.-X. Qin, and C. D. Roberts, Phys. Rev. D 93, 096010 (2016).

[18] H. J. Munczek, Phys. Rev. D 52, 4736 (1995).

[19] A. Bender, C. D. Roberts, and L. Von Smekal, Phys. Lett. B 380, 7 (1996).

[20] J. C. Ward, Phys. Rev. 78, 182 (1950).

[21] H. S. Green, Proc. Phys. Soc. London Sect. A 66, 873 (1953).

[22] Y. Takahashi, Nuovo Cimento 6, 371 (1957).

[23] L. Chang and C. D. Roberts, Phys. Rev. Lett. 103, 081601 (2009).

[24] S.-X. Qin and C. D. Roberts, Chin. Phys. Lett. 38, 071201 (2021).

[25] L. Chang and M. Ding, Phys. Rev. D 103, 074001 (2021).

[26] L. Chang, Y.-X. Liu, and C. D. Roberts, Phys. Rev. Lett. 106, 072001 (2011).

[27] A. Bashir, R. Bermudez, L. Chang, and C. D. Roberts, Phys. Rev. C 85, 045205 (2012).

[28] D. J. Wilson, I. C. Clet, L. Chang, and C. D. Roberts, Phys. Rev. C 85, 025205 (2012).

[29] K. Raya, L. X. Gutiérrez, and A. Bashir, Few Body Syst. 59, 89 (2018).

[30] K. Raya, L. X. Gutiérrez-Guerrero, A. Bashir, L. Chang, Z.-F. Cui, Y. Lu, C. D. Roberts, and J. Segovia, arXiv: 2108.02306. 
[31] L. Albino, A. Bashir, L. X. G. Guerrero, B. E. Bennich, and E. Rojas, Phys. Rev. D 100, 054028 (2019).

[32] M. Atif Sultan, K. Raya, F. Akram, A. Bashir, and B. Masud, Phys. Rev. D 103, 054036 (2021).

[33] S.-X. Qin, L. Chang, Y.-X. Liu, C. D. Roberts, and D. J. Wilson, Phys. Rev. C 84, 042202 (2011).

[34] P. Maris and P.C. Tandy, Phys. Rev. C 60, 055214 (1999).

[35] M. Ding, K. Raya, D. Binosi, L. Chang, C. D. Roberts, and S. M. Schmidt, Phys. Rev. D 101, 054014 (2020).

[36] M. Ding, K. Raya, A. Bashir, D. Binosi, L. Chang, M. Chen, and C. D. Roberts, Phys. Rev. D 99, 014014 (2019).

[37] Y.-B. Dai, C.-S. Huang, and D.-S. Liu, Phys. Rev. D 43, 1717 (1991).

[38] L. X. Gutierrez-Guerrero, A. Bashir, I. C. Clet, and C. D. Roberts, Phys. Rev. C 81, 065202 (2010).

[39] H. L. L. Roberts, C. D. Roberts, A. Bashir, L. X. GutierrezGuerrero, and P. C. Tandy, Phys. Rev. C 82, 065202 (2010).

[40] P.-L. Yin, Z.-F. Cui, C. D. Roberts, and J. Segovia, Eur. Phys. J. C 81, 327 (2021).

[41] L. X. Gutiérrez-Guerrero, A. Bashir, M. A. Bedolla, and E. Santopinto, Phys. Rev. D 100, 114032 (2019).

[42] P.-L. Yin, C. Chen, G. Krein, C. D. Roberts, J. Segovia, and S.-S. Xu, Phys. Rev. D 100, 034008 (2019).

[43] C. Chen, L. Chang, C. D. Roberts, S. Wan, and D. J. Wilson, Few Body Syst. 53, 293 (2012).

[44] M. A. Bedolla, J. Ferretti, C. D. Roberts, and E. Santopinto, Eur. Phys. J. C 80, 1004 (2020).

[45] K. Raya, M. A. Bedolla, J. J. Cobos-Martínez, and A. Bashir, Few Body Syst. 59, 133 (2018).

[46] M. A. Bedolla, K. Raya, J. J. Cobos-Martínez, and A. Bashir, Phys. Rev. D 93, 094025 (2016).

[47] J. Segovia, B. El-Bennich, E. Rojas, I. C. Clet, C. D. Roberts, S.-S. Xu, and H.-S. Zong, Phys. Rev. Lett. 115, 171801 (2015).
[48] J. Segovia, I. C. Cloët, C. D. Roberts, and S. M. Schmidt, Few Body Syst. 55, 1185 (2014).

[49] D. Ebert, T. Feldmann, and H. Reinhardt, Phys. Lett. B 388 , 154 (1996).

[50] F. E. Serna, B. El-Bennich, and G. Krein, Phys. Rev. D 96, 014013 (2017).

[51] H. L. L. Roberts, A. Bashir, L. X. Gutierrez-Guerrero, C. D. Roberts, and D. J. Wilson, Phys. Rev. C 83, 065206 (2011).

[52] P. Zyla et al. (Particle Data Group), Prog. Theor. Exp. Phys. 2020, 083C01 (2020).

[53] Y. Lu, C. Chen, C. D. Roberts, J. Segovia, S.-S. Xu, and H.-S. Zong, Phys. Rev. C 96, 015208 (2017).

[54] P. Maris and P. C. Tandy, Phys. Rev. C 65, 045211 (2002).

[55] G. Eichmann, C. S. Fischer, and R. Williams, Phys. Rev. D 101, 054015 (2020).

[56] A. S. Miramontes, H. Sanchis Alepuz, and R. Alkofer, Phys. Rev. D 103, 116006 (2021).

[57] Y.-Z. Xu, S. Chen, Z.-Q. Yao, D. Binosi, Z.-F. Cui, and C. D. Roberts, arXiv:2107.03488.

[58] P. Maris and P. C. Tandy, Phys. Rev. C 61, 045202 (2000).

[59] R. Bermudez, L. Albino, L. X. Gutiérrez-Guerrero, M. E. Tejeda-Yeomans, and A. Bashir, Phys. Rev. D 95, 034041 (2017).

[60] C. D. Roberts, Nucl. Phys. A605, 475 (1996).

[61] Y.-Z. Xu, D. Binosi, Z.-F. Cui, B.-L. Li, C. D. Roberts, S.-S. Xu, and H. S. Zong, Phys. Rev. D 100, 114038 (2019).

[62] B. Owen, W. Kamleh, D. Leinweber, B. Menadue, and S. Mahbub, Phys. Rev. D 91, 074503 (2015).

[63] P. Ball and V. M. Braun, Phys. Rev. D 54, 2182 (1996).

[64] F. Gao, L. Chang, Y.-X. Liu, C. D. Roberts, and S. M. Schmidt, Phys. Rev. D 90, 014011 (2014).

[65] G. P. Lepage and S. J. Brodsky, Phys. Rev. D 22, 2157 (1980).

[66] G. Eichmann, I. C. Clet, R. Alkofer, A. Krassnigg, and C. D. Roberts, Phys. Rev. C 79, 012202 (2009). 